\title{
PERFIL DOS USUÁRIOS DO HIPERDIA DE TRÊS UNIDADES BÁSICAS DE SAÚDE DO SUL DO BRASILa
}

\author{
Lílian M oura de LI M A ${ }^{b}$, E da SCHWART Zc , R osani M anfrin M U N I Z ${ }^{b}$,

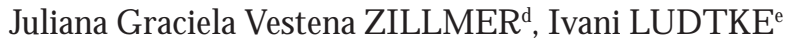

\begin{abstract}
RESUM 0
O bjetivou-se descrever o perfil dos hipertensos e diabéticos cadastrados no sistema HiperDia do M inistério da Saúde em três unidades básicas do município de Pelotas, na região Sul do Rio G rande do Sul. T rata-se de um estudo transver sal com abordagem quantitativa. U tilizou-se como instrumento de pesquisa um for mulário baseado na ficha de cadastro do sistema HiperDia, que for nece informações importantes sobre a população atendida. Os dados coletados foram digitados no programa E pi-info 6.04 e analisados no software STATA 9.0. A população avaliada no estudo é predominantemente feminina, na faixa etária acima dos 50 anos. Os fatores de risco mais presentes foram o sobrepeso, o hábito de vida sedentário, a hereditariedade e como complicações crônicas as cardiovasculares. Concluiu-se que o perfil dos cadastrados é semel hante ao encontrado no banco de dados nacional do SisH iperDia.
\end{abstract}

D escritores: Saúde pública. Hipertensão. Diabetes M ellitus.

\section{RESUMEN}

E I objetivo fue describir el perfil de pacientes hipertensos y diabéticos inscritos en el sistema $\mathrm{H}$ iperD ia del M inisterio de la Salud en tres unidades básicas de P elotas en el sur de R io G rande do Sul, B rasil. E studio transversal con abordaje cuantitativo; seutilizó como her ramienta de investigación un formulario basado en el registro del sistema $\mathrm{H}$ iperD ia, este si stema proporciona informaciones importantes sobre la población atendida. $L$ os datos recolectados fuer on ingresados en el programa E pi- Info 6,04 y analizados en STA TA 9.0. L a población evaluada es predominantementefemenina, del grupo etario mayor de 50 años. L os factores de riesgo más presentes fueron sobrepeso, estilo de vida sedentario, herencia y complicaciones crónicas cardiovasculares. Se concluyó que el perfil de inscritos es similar a lo encontrado en la base de datos nacional deSisH iperD ia.

D escriptores: Salud pública. H ipertensión. D iabetes M ellitus.

Título: P erfil de los usuarios registrados en el H iperD ia de tres unidades básicas de salud del Sur de B rasil.

\section{ABST RACT}

This study aims to describe the profile of hypertensive and diabetic patients from three primary health units in the city of P el otas, southern R io G rande do Sul, B razil, that are registered in the M inistry of $\mathrm{H}$ ealth's $\mathrm{H}$ iperD ia system. This is a crosssectional study with a quantitative approach. A form based on the registration form of the $\mathrm{H}$ iper $\mathrm{D}$ ia system was used as a research instrument. T his system provides important information about the population served. The collected data were entered into the E pi-Info 6.04 computer program and analyzed in the STATA 9.0 software The subjects of the study are predominantly female, aged above 50 years. P redominant risk factors are overw eight, sedentary lifestyle, her edity and chronic cardi ovascul ar complications. We concluded that the profile of thesubjects of the study is similar to that of SisH iperD ia's national database

D escriptors: P ublic health. $\mathrm{H}$ ypertension. D iabetes $\mathrm{M}$ ellitus.

T itle: P rofile of $\mathrm{H}$ iper D ia system users from three primary healthcare units in southern B razil.

\footnotetext{
a Artigo originado do trabal ho de conclusão do Curso de E specialização M ultiprofissional em E stratégia de Saúde da F amília, apresentado em 2009 na Faculdade de Enfermagem da Universidade F ederal de Pelotas (U F Pel).

' E special ista em Estratégia de Saúde da F amília, M estranda pelo Prog rama de Pós-G raduação em Enfer magem da Faculdade de Enfer magem da U F Pel, D ocente da F aculdade de Enfermagem da Universidade A nhanguera, Pelotas, Rio G rande do Sul, Brasil.

' D outora em Enfermagem, D ocente da Faculdade de Enfermagem da U F Pel, Pelotas, Rio G rande do Sul, Brasil.

d M estre em Enfermagem, D outoranda pelo Programa de Pós-Graduação em Enfermagem da U niversidade Federal de Santa Catarina (UFSC), Florianópolis, Santa Catarina, Brasil.

e Especialista em E stratégia de Saúde da F amília, E nfer meira da E stratégia da Saúde da Família (E SF ) de Pelotas, Rio G rande do Sul, Brasil.
} 


\section{INT RODUÇÃO}

As transformações sofridas pela população mundial, no último século, no que diz respeito à alimentação, à expectativa de vida e às causas de morte redefiniram o perfil de suscetibilidade às doenças, ou seja, as mudanças socioeconômicas e culturais refletiram em maus hábitos alimentares, sedentarismo e consequente sobrepeso. A união destes fatores ao envel hecimento populacional favoreceu a ocorrência das condições crônicas como a hiper tensão arterial e 0 diabetes ${ }^{(1)}$.

Com isso, estas doenças passaram a ocupar lugar de destaque entre os problemas de saúde pública, visto que, seus portadores necessitam de um acompanhamento contínuo e cuidadoso, caso contrário, podem evoluir para complicações crônicas consequentes da doença. Estas últimas são, em sua maioria, graves e geram grande transtorno à sociedade em geral, não apenas pelo ônus financeiro de seu tratamento, mas também pela redução da qualidade de vida e produtividade do indivíduo acometido(2).

Com base no exposto, compreende-se a necessidade do desenvolvimento de programas de atenção básica, destinados à prevenção, identificação e acompanhamento dos casos de hipertensão arterial e diabetes. Para tanto, o M inistério da Saúde desenvolveu o plano de reorganização da atenção à hipertensão arterial sistêmica (HAS) e ao diabetes melito (DM ), no ano de 2000, e com ele iniciou-se a inscrição nacional destes indivíduos no sistema de cadastramento e acompanhamento de Hipertensos e D iabéticos (H iperD ia) e programa nacional de assistência farmacêutica para a HAS e o DM (3).

E ntretanto, para que as ações estipuladas neste plano sejam efetivas é necessário que elas acompanhem as transformações da demanda atendida. E esta informação é obtida pela base de dados do sistema HiperDia e do desenvolvimento e publicação de estudos que descrevam as características da população de diabéticos e hipertensos.

Observou-se em publicações recentes sobre o perfil dos cadastrados no sistema H iperDia, que estes eram em sua maioria acometidos por hipertensão arterial, estavam na faixa etária acima de 50 anos e eram do sexo feminino ${ }^{(4,5)}$. Verificando-se a base de dados do Departamento de Informática do Sistema Ú nico de Saúde (DATASUS) para todos os estados brasileiros, constatou-se que o número de usuários do sexo feminino se sobressaiu em rela- ção ao masculino e que a prevalência de hipertensão arterial se mantém superior as de diabetes e hipertensão mais diabetes ${ }^{(6)}$.

A base de dados do sistema HiperDia é extensa e permite realizar um diagnóstico da população acometida por diabetes e hipertensão arterial, estratificada por estado e cidade. Entretanto, pode-se verificar que as unidades básicas de saúde, muitas vezes, não têm conhecimento do perfil de seus usuários e, por esse motivo, não abordam esta população com efetividade.

A lém disso, verificou-se que existem poucas publicações científicas, envolvendo o perfil da população cadastrada no sistema HiperDia. D essa forma, este estudo vem acrescentar informações importantes sobre as características deste grupo populacional na cidade de Pel otas, R io G rande do Sul, e tem por objetivo descrever o perfil dos hipertensos e diabéticos cadastrados no sistema H iperDia do M inistério da Saúde em três unidades básicas deste município(7).

\section{MÉT ODO}

Realizou-se estudo descritivo, de corte transversal, em três unidades básicas de saúde da cidade de Pelotas, utilizando-se dados secundários, coletados a partir da segunda via das fichas de cadastro do sistema HiperDia do M inistério da Saúde, para caracterizar a população alvo da pesquisa.

Por se tratar de um estudo de demanda, a amostra foi constituída pelos usuários diabéticos e hipertensos cadastrados no sistema H iperD ia por intermédio de uma das U nidades Básicas de Saúde (UBS) envolvidas no estudo. N ão foram adotados critérios de exclusão.

As variáveis independentes incluídas na análise foram: sexo; idade, distribuída em décadas; cor da pele classificada de acordo com a percepção do paciente como branca e preta ou outra; escolaridade (não alfabetizado, primeiro grau incompleto, primeiro grau completo, segundo grau incompleto, segundo grau completo e ensino superior completo); situação conjugal (com companheiro, sem companheiro, vive só); índice de massa corporal, pela classificação da Organização M undial da Saúde (OM S) em peso normal (I M C $\geq 18,5 \mathrm{Kg} / \mathrm{m}^{2} \mathrm{e}<$ $\left.25 \mathrm{Kg} / \mathrm{m}^{2}\right)$, sobrepeso (IM C $\geq 25 \mathrm{~kg} / \mathrm{m}^{2} \mathrm{e}<30 \mathrm{~kg} /$ $\left.\mathrm{m}^{2}\right)$ e obesidade $\left(\mathrm{IMC} \geq 30 \mathrm{Kg} / \mathrm{m}^{2}\right)^{(8)}$; presença de hipertensão, diabetes, antecedentes familiares, tabagismo (na ficha de cadastramento do HiperDia consta apenas a utilização de tabaco no momento 
do registro, desconsiderando tabagismo prévio), sedentarismo, infarto, outra cardiopatia, acidente vascular cer ebral (AVC), doença renal, pé diabético e amputação por diabete, categorizados como sim e não.

0 instrumento de coleta de dados foi um questionário pré-codificado, elaborado a partir da ficha de cadastramento do sistema HiperDia. Para testar este instrumento, foi realizado um estudo piloto, em uma unidade básica de saúde do município de Pelotas, no mês de setembro de 2008, com posteriores ajustes do questionário e construção de um manual de preenchimento para nortear os pesquisadores durante a coleta dos dados. E sta se deu no período de 06 de outubro a 14 de novembro de 2008.

Com a totalidade dos dados coletados, foi realizada a codificação dos instrumentos e a construção do banco de dados no E pi I nfo 6.04, observando dupla digitação. Posteriormente, foi real izada a análise descritiva e estratificada dos dados com 0 software ST ATA 9.0.
0 presente projeto está vinculado à pesquisa "Avaliação de Serviços em U nidades Básicas T radicionais e com Estratégia de Saúde da Família: Diagnóstico da Situação de Pelotas e Região", o qual foi encaminhado ao Comitê de Ética em Pesquisa e recebeu parecer favorável sob ofício $n-025 /$ 08. Para o estudo, não foi necessário utilizar-se termo de consentimento livree esclarecido, por se tratar de coleta de dados secundários.

\section{RESULT ADOS}

F oram avaliadas, neste estudo, 1.193 fichas de inscrição do sistema HiperDia. Observa-se, nas Tabelas 1 e 2, a estratificação do perfil dos usuários dentre as variações de diagnóstico que os levaram ao cadastramento no sistema HiperDia. Observou-se maior ocorrência de hipertensos com $63 \%$ dos indivíduos, seguidos pelos hipertensos e concomitantemente diabéticos com $31,3 \%$, enquanto que apenas 2,5\% for am diagnosticados como exclusivamente diabéticos.

T abela 1 - Caracterização sócio-demográfica dos usuários cadastrados no HiperDia, por doença de base. Pelotas, RS, 2009 ( $\mathrm{N}=1193)$.

\begin{tabular}{|c|c|c|c|c|c|c|}
\hline \multirow{2}{*}{ Características } & \multicolumn{2}{|c|}{ Hipertensos $(\mathrm{n}=752)$} & \multicolumn{2}{|c|}{ Diabéticos $(n=30)$} & \multicolumn{2}{|c|}{ Hipertensos e diabéticos $(n=373)$} \\
\hline & $\mathbf{n}$ & $\%$ & $\mathbf{n}$ & $\%$ & $\mathbf{n}$ & $\%$ \\
\hline \multicolumn{7}{|l|}{ Sexo } \\
\hline Feminino & 517 & 69,6 & 14 & 46,7 & 268 & 73,0 \\
\hline$M$ asculino & 226 & 30,4 & 16 & 53,3 & 99 & 27,0 \\
\hline \multicolumn{7}{|l|}{ Idade } \\
\hline 20 a 29 anos & 15 & 2,1 & - & - & 5 & 1,4 \\
\hline 30 a 39 anos & 44 & 6,3 & 2 & 8,0 & 14 & 4,0 \\
\hline 40 a 49 anos & 130 & 18,5 & 6 & 24,0 & 50 & 14,2 \\
\hline 50 a 59 anos & 184 & 26,1 & 9 & 36,0 & 91 & 25,8 \\
\hline 60 a 69 anos & 166 & 23,6 & 4 & 16,0 & 113 & 31,9 \\
\hline$\geq 70$ anos & 165 & 23,4 & 4 & 16,0 & 80 & 22,7 \\
\hline \multicolumn{7}{|l|}{ Cor da pele } \\
\hline Branca & 494 & 69,0 & 23 & 76,7 & 250 & 69,4 \\
\hline Preta & 202 & 28,2 & 6 & 20,0 & 110 & 30,6 \\
\hline A marela & 4 & 0,6 & 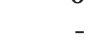 & & $1+0$ & \\
\hline Parda & 12 & 1,6 & 1 & 3,3 & - & - \\
\hline Indígena & 4 & 0,6 & - & & - & - \\
\hline \multicolumn{7}{|l|}{ E scolaridade } \\
\hline N ão sabe ler/ escrever & 102 & 14,4 & 5 & 17,2 & 51 & 14,6 \\
\hline 1o grau incompleto & 484 & 68,1 & 13 & 44,8 & 223 & 63,9 \\
\hline 1o grau completo & 77 & 10,8 & 3 & 10,3 & 45 & 12,9 \\
\hline 20 grau completo & 42 & 5,9 & 6 & 20,7 & 24 & 6,9 \\
\hline Superior completo & 6 & 0,8 & 2 & 7,0 & 6 & 1,7 \\
\hline \multicolumn{7}{|l|}{ Situação conjugal* } \\
\hline Com companheiro & 456 & 69,5 & 21 & 84,0 & 222 & 67,7 \\
\hline Sem companheiro & 180 & 27,4 & 4 & 16,0 & 100 & 30,5 \\
\hline Vive só & 20 & 3,1 & - & - & 6 & 1,8 \\
\hline
\end{tabular}

*A variável "situação conjugal" apresentou o maior número de ignorados (184).

Fonte: Fichas de cadastramento de usuários no H iperDia, arquivadas nas três U nidades Básicas de Saúde de Pel otas incluídas no estudo. 
$\mathrm{N} \mathrm{a} \mathrm{T}$ abela 1 observa-se a distribuição das características sócio-demográficas dos usuários cadastrados no HiperDia por patologia de base.

Os diagnosticados com HAS ( $n=752$ ) são, na amostra analisada, em sua maioria usuários do sexo feminino, $69,6 \%$, e estão agrupados em maior número na faixa etária de 50 a 59 anos com 26,1\%. Do total de usuários hipertensos, $69 \%$ referiram ser da cor branca, $69,0 \%$ relataram ter cursado 0 primeiro grau incompleto e $69,5 \%$ mencionaram viver com companheiro.

De acordo com os dados dos usuários cadastrados com DM $(n=30)$, houve um discreto predomínio no número de homens $53,3 \%$, a fai- xa etária mais frequente foi de 50 a 59 anos, 36\% E $m$ relação à cor da pele, o predomínio foi da raça branca com $76,7 \%$. Os cadastrados referiram em $44,8 \%$ cursaram o primeiro grau incompleto e $84,0 \%$ viverem com companheiro.

O grupo composto pelos indivíduos afetados pelas patologias DM e HAS $(n=373)$, foi composto por $73 \%$ de indivíduos do sexo feminino, a faixa etária predominante entre 60 a 69 anos 31,9\%, cor da pele referida como branca com $69,4 \%$. A escolaridade predominante no grupo foi primeiro grau incompleto com $63,9 \%$ dos entrevistados e $67,7 \%$ deles referiram viver com companheiro.

T abela 2 - Fatores de risco e complicações crônicas dos usuários cadastrados no HiperDia por doença de base. Pelotas, RS, 2009 ( $N=1193)$.

\begin{tabular}{|c|c|c|c|c|c|c|}
\hline \multirow{2}{*}{ Características } & \multicolumn{2}{|c|}{ Hipertensos $(n=752)$} & \multicolumn{2}{|c|}{ D iabéticos $(n=30)$} & \multicolumn{2}{|c|}{$\begin{array}{l}\text { Hipertensos e diabéticos } \\
(\mathrm{n}=373)\end{array}$} \\
\hline & $\mathbf{n}$ & $\%$ & $\mathbf{n}$ & $\%$ & $\mathbf{n}$ & $\%$ \\
\hline \multicolumn{7}{|c|}{ Índice de M assa Corporal* } \\
\hline N ormal & 198 & 27,5 & 9 & 30,0 & 82 & 22,9 \\
\hline Sobrepeso & 298 & 41,4 & 14 & 46,7 & 149 & 41,6 \\
\hline Obesidade & 224 & 31,1 & 7 & 23,3 & 127 & 35,5 \\
\hline \multicolumn{7}{|c|}{$\begin{array}{l}\text { A ntecedentes familiares } \\
\text { cardiovasculares }\end{array}$} \\
\hline N ão & 269 & 36,5 & 14 & 46,7 & 114 & 31,2 \\
\hline Sim & 468 & 63,5 & 16 & 53,3 & 251 & 68,8 \\
\hline \multicolumn{7}{|l|}{ T abagismo } \\
\hline Não & 545 & 73,1 & 25 & 86,2 & 291 & 80,4 \\
\hline Sim & 201 & 26,9 & 4 & 13,8 & 71 & 19,6 \\
\hline \multicolumn{7}{|l|}{ Sedentarismo } \\
\hline Não & 275 & 37,0 & 11 & 37,9 & 152 & 42,0 \\
\hline Sim & 469 & 63,0 & 18 & 62,1 & 210 & 58,0 \\
\hline \multicolumn{7}{|c|}{ Infarto Agudo do M iocárdio } \\
\hline Não & 686 & 92,1 & 27 & 93,1 & 320 & 88,4 \\
\hline Sim & 59 & 7,9 & 2 & 6,9 & 42 & 11,6 \\
\hline \multicolumn{7}{|c|}{ O utra cardiopatia } \\
\hline Não & 610 & 82,4 & 27 & 93,1 & 272 & 75,8 \\
\hline Sim & 130 & 17,6 & 2 & 6,9 & 87 & 24,2 \\
\hline \multicolumn{7}{|c|}{ A cidente Vascular Cerebral } \\
\hline N ão & 691 & 93,5 & 29 & 100 & 320 & 88,4 \\
\hline Sim & 48 & 6,5 & - & - & 42 & 11,6 \\
\hline \multicolumn{7}{|l|}{ D oença Renal } \\
\hline N ão & 718 & 96,3 & 28 & 96,5 & 339 & 93,9 \\
\hline Sim & 28 & 3,7 & 1 & 3,5 & 22 & 6,1 \\
\hline \multicolumn{7}{|l|}{ Pé diabético } \\
\hline N ão & 745 & 100 & 27 & 93,1 & 352 & 97,5 \\
\hline Sim & & & 2 & 6,9 & 9 & 2,5 \\
\hline \multicolumn{7}{|c|}{ Amputação por diabete } \\
\hline Não & 745 & 100 & 28 & 96,5 & 357 & 98,3 \\
\hline $\mathrm{Sim}$ & - & - & 1 & 3,5 & 6 & 1,7 \\
\hline
\end{tabular}

* Normal: $\geq 18,5 \mathrm{Kg} / \mathrm{m}^{2}$ e $<25 \mathrm{Kg} / \mathrm{m}^{2}$; Sobrepeso: $\geq 25 \mathrm{~kg} / \mathrm{m}^{2} \mathrm{e}<30 \mathrm{~kg} / \mathrm{m}^{2}$; O besidade: $\geq 30 \mathrm{Kg} / \mathrm{m}^{2}$.

Fonte: Fichas de cadastramento de usuários no HiperDia, arquivadas nas três U nidades Básicas de Saúde de Pelotas incluídas no estudo. 
$\mathrm{Na}$ T abela 2 verifica-se a distribuição dos fatores de risco e complicações crônicas dos cadastrados no Hiperdia por patologia de base. Sendo que o grupo de hipertensos ( $n=752$ ) apresentava $41,4 \%$ de indivíduos com sobrepeso; $63,5 \%$ com antecedentes familiares cardiovasculares; $26,9 \%$ de tabagistas no momento do cadastro; e 63,0\% admitiram ser sedentários. $D$ as complicações decorrentes da hipertensão, 7,9\% referiam ter sofrido infarto agudo do miocár dio (IAM ), 17,6\% outras cardiopatias, 6,5\% AVC e 3,7\% doença renal.

D entre os cadastrados com DM $(n=30) 46,7 \%$ apresentavam sobrepeso; a presença de antecedentes familiares cardiovasculares foi referida por $53,3 \%$ desta amostra; $13,8 \%$ eram tabagistas no momento do cadastro; $62,1 \%$ admitiram ser sedentários; e 6,9\% referiram ter sofrido de IAM, outras cardiopatias e pé diabético; ficando a proporção de 3,5\% dos cadastrados com as complicações de doença renal e amputação em decorrência do $D M$.

Dentre os diagnosticados com H AS eD M ( $n=$ 373), 41,6\% apresentavam sobrepeso, $68,8 \%$ referiram ter antecedentes familiares cardiovasculares, 58,0\% admitiram ser sedentários e 19,6\% eram fumantes no momento do cadastro. Das complicações questionadas na ficha de cadastro do sistema $H$ iperDia, foram referidas em ordem decrescente: outras cardiopatias com 24,2\%; I AM e AVC com $11,6 \%$; doença renal com 6,1\%; pé diabético 2,5\%; e amputação por diabetes $1,7 \%$.

\section{DISCUSSÃO}

As limitações deste estudo estiveram relacionadas à utilização de dados secundários, pois estes, muitas vezes, foram de difícil interpretação devido a problemas no preenchimento da ficha de cadastramento no sistema H iperDia, tais como, dados ilegíveis e informações incompletas.

$\mathrm{Na}$ amostra estudada, houve predomínio de usuários do sexo feminino, o que vai ao encontro dos dados publicados no site do DATASU $S^{(6)}$, em relação a todas as regiões do país. E m estudos sobre o perfil de usuários acompanhados pelo sistema HiperDia, foram encontrados resultados semelhantes em relação à distribuição entre os sexos, sendo justificada a superioridade feminina dos cadastros, em virtude da maior procura das muIheres pelo sistemas de saúde, da sua maior percepção a respeito do estado de saúde-doença e por apresentarem maior tendência ao autocuidado e a busca de auxílio médico para si e para seus familiares $(5,9,10)$

A população estudada concentrou-se na faixa de idade acima dos 50 anos, o que vai ao encontro da situação das doenças crônicas não transmissíveis (DCNT) no país ${ }^{(6)}$. A hipertensão arterial sistêmica e o diabetes melito têm sua prevalência aumentada em indivíduos acima dos cinquenta anos de idade, além disso, deve-se considerar o envelhecimento populacional brasileiro(4).

Em se tratando do nível de escolaridade dos cadastrados, verifica-se a maior proporção de indivíduos com primeiro grau incompleto, o que é um fator preocupante em se tratando de pacientes crônicos que necessitam manter um nível de cuidado el aborado em relação a medicamentos e restrições alimentares. A baixa escolaridade dificulta o processo de ensino aprendizagem, sendo que estes indivíduos necessitam de atenção especial e adequação das ações educativas do profissional de saúde para que haja melhor compreensão dos cuidados necessários para o controle da doença crônica e a prevenção de complicações ${ }^{(11,12)}$. A credita-se que a co-responsabilização pelo tratamento da doença entre 0 usuário e a equipe de saúde, assim como a maior frequência nas interações entre estes sujeitos, proporciona a superação das dificuldades relacionadas à manutenção dos hábitos necessários para o controle da doença ${ }^{(11-13)}$.

Quanto aos fatores de risco, observou-se que os participantes estiveram concentrados no índice de massa corporal de sobrepeso $41,7 \%$, os antecedentes familiares estiveram presentes em 65,3\% dos cadastrados, $24,3 \%$ eram tabagistas e $61 \%$ referiram ser sedentários. E m estudo realizado a partir do SisHiperDia/ M S ( $n=387.754)$ foram identificados resultados similares, assim como no estudo realizado com pacientes diabéticos cadastrados no H iperD ia ( $n=7.938)$, na cidade de Cuiabá, que encontrou 43\% de usuários sedentários. Estes autores remetem à provável associação desta porcentagem com a prevalência das co-morbidades observadas entre os pacientes do estudo ${ }^{(14,15)}$. Os fatores relacionados aos hábitos de vida, como a obesidade e o sedentarismo, têm influencia dir eta no estabel ecimento e no controle da hipertensão e do diabetes, e têm sido considerados fatores de risco para a mortalidade prematura ${ }^{(16)}$.

D essa forma, com base nos resultados deste estudo fica evidenciada a relevância do estabelecimento de atividades educativas com a popula- 
ção estudada, com o objetivo de estimular a prática de atividade física regular e a ingestão calórica adequada.

D as complicações crônicas relacionadas na ficha de cadastro, na presente amostra, a mais frequente foi outra cardiopatia (19,3\%), seguida de I AM $(9,1 \%)$, AVC $(8,1 \%)$ e doença renal $(4,6 \%)$. As complicações vasculares decorrentes da HAS originam lesões em órgãos como coração, cér ebro e rins. Cerca de $70 \%$ dos indivíduos em programa de hemodiálise têm como causa da lesão primária a HAS, e nos casos de associação desta doença ao diabetes, as lesões renais costumam ser mais precoces e intensas ${ }^{(2)}$.

As complicações de pé diabético e a amputação por diabetes estiveram em pequeno número. 0 mesmo resultado foi encontrado em pesquisa realizada com diabéticos na cidade de Fortaleza, 0 que, segundo 0 autor, pode estar relacionado a resultados de campanhas educativas realizadas, nesta localidade, para os cuidados com os pés no diabetes ${ }^{(5)}$. A realização de intervenções com estes sujeitos têm impacto significativo na redução das complicações do DM, inclusive na ocorrência do pé diabético. 0 que foi evidenciado em um estudo desenvolvido na cidade de R io Branco-Acre, no qual, foi investigado o conhecimento dos pacientes diabéticos sobre as medidas de cuidados preventivos do pé diabético $(n=109)$, antes da atividade educativa e após em média 84,2 (D P $=30,4$ ) dias da sua realização. Foi identificado que no préteste o conhecimento dos pacientes era inferior a mediana em $38,5 \%$, e no pós-teste este resultado foi reduzido para 3,7\% dos entrevistados abaixo da mediada, o que demonstra a efetividade da realização deste tipo de inter venção na atenção básica à saúde ${ }^{(13)}$.

\section{CONCLUSÕES}

As condições crônicas HAS e DM representam um problema de saúde pública em virtude das complicações agudas e crônicas delas decorrentes, havendo necessidade da realização de estudos que busquem descrever esta população, com vistas a oferecer subsídios para o planejamento de ações que possam melhorar a qualidade de vida destes indivíduos, retardando o aparecimento de complicações crônicas.

E m conclusão os achados do estudo evidenciam que a população de diabéticos e hipertensos cadastrada nas três U BS's estudadas, tem o perfil semelhante ao dos cadastrados em âmbito nacional. Há maior número de mulheres, com diagnóstico de HAS, na faixa etária acima de cinquenta anos, que moram com companheiro e cursaram o primeiro grau incompleto. Em relação às complicações crônicas decorrentes das doenças, as mais comuns foram às cardiovasculares e os fatores de risco estiveram relacionados aos hábitos de vida sedentários, ao sobrepeso e a obesidade.

\section{REFERÊ NCIAS}

1 Toscano CM. As campanhas nacionais para detecção das doenças crônicas não-transmissíveis: diabetes e hipertensão arterial. Ciênc Saúde Colet [ Internet] . 2004 [ citado 2010 mar 15] ;9(2):885-95. Disponível em: http:// www.scielo.br/pdf/csc/v9n4/ alov9n4.pdf.

2 M inistério da Saúde (BR). Plano de reorganização da atenção a hipertensão arterial e ao diabetes mellitus: manual de hipertensão arterial e diabetes. Brasília (D F); 2002.

3 M inistério da Saúde (BR). Avaliação do plano de reorganização da hipertensão arterial e ao diabetes mellitus no Brasil. Brasília (DF); 2004.

4 H enrique N N, Costa PS, Vileti JL, Corrêa M CM , Carval ho EC. H ipertensão ar terial e diabetes mell itus: um estudo sobre os programas de atenção básica. Rev Enferm UER] [ Internet] . 2008 [ citado 2010 mar $20]$;16(2):168-73. Disponível em: http:/ / www.facenf. uerj.br/ v16n2/ v16n2a05.pdf.

5 M oreira T, Silva EA, Oliveira CJ, A breu RN DC, Vasconcelos F F. Caracterização dos pacientes acompanhados pel o prog rama $\mathrm{H}$ iper D ia em uma unidade básica de saúde da família em Fortaleza. N ursing [ I nternet] . 2009 [ citado 2010 abr 04];11(130):137-42. Disponível em: http:/ / www.nursing.com.br/ paper. php? $\mathrm{p}=440$.

6 M inistério da Saúde (BR), D epartamento de Informática do Sistema Ú nico de Saúde. SisH iperDia [ Internet]. Brasília (DF); 2009 [ citado 2009 maio 20] . Disponível em: http:/ / hiperdia.datasus.gov.br/ .

7 Lima L M . Perfil dos usuários cadastrados no programa Hiperdia de três unidades básicas de saúde de Pelotas/RS [ monografia]. Pelotas: Faculdade de Enfer magem, U niversidade Federal de Pelotas; 2009. 
$8 \mathrm{~W}$ orld $\mathrm{H}$ ealth Organization. $O$ besity: preventing and manging the global epidemic: report of a W HO consultation on obesity. G eneva; 2000.

9 Zaitune M PA, Barros M BA, César CLG, Carandina $L, G$ oldbaum M. Hipertensão arterial em idosos: prevalência, fatores associados e práticas de controle no M unicípio de Campinas. Cad Saúde Pública [ Internet] . 2006 [ citado 2010 jun 10];22(2):285-94. Disponível: http:/ / www.scielosp.org/ pdf/ csp/ v22 n2/ 06.pdf.

10 Contiero AP, Pozati M PS, Challouts RI, Carreira $L, M$ arcon SS. I doso com hipertensão arterial: dificuldades de acompanhamento na Estratégia Saúde da Família. Rev G aúcha Enferm [ Internet] . 2009 [ citado 2010 jun 20];30(1):62-70. Disponível em: http:/ / www.seer.ufrgs.br/ index.php/ RevistaG aucha deE nfermagem/ article/ view/ 4227/ 6564.

11 M orais G F C, Soares M J G O, Costa M M L, Santos IBC. 0 diabético diante do tratamento, fatores de risco e complicações crônicas. Rev Enferm UER] [ Internet] . 2009 [ citado 2011 abr 6] ;17(2):240-5. Disponível em: http:/ / www.facenf.uerj.br/v17n2/ v17 n2a18.pdf.

12 Laurindo M C, Recco DC, Roberti D B, Rodrigues CDS. Conhecimento das pessoas diabéticas acerca dos cuidados com os pés. A rq Ciênc Saúde [ I nter net] . 2005
[ citado 2011 abr 5];12(2):80-4. D isponível em: http:/ / files.bvs.br/ upload/ S/ 0100-0233/ 2009/ v33n3/ a005.pdf.

13 Cosson I CO, N ey-O liveira F, A dan LF. Avaliação do conhecimento de medidas preventivas do pé diabético em pacientes de Rio Branco, Acre. Arq Bras E ndocrinol M etab [ I nternet] . 2005 [ citado 2011 abr 06] ;49(4):548-56. Disponível em: http:/ / www.scielo. br/pdf/ abem/ v49n4/ a13v49n4.pdf.

14 Boing $A C$, Boing AF. Hipertensão arterial sistêmica: 0 que nos dizem os sistemas brasileiros de cadastramento e informações em saúde. Rev Bras Hipertens [ I nternet] . 2007 [ citado 2010 jul 01] ;14(2):848. D isponível: http:/ / departamentos.cardiol.br/ dha/ revista/ 14-2/ 06-hipertensao.pdf.

15 Ferreira CA, Ferreira M G. Características epidemiológicas de pacientes diabéticos da rede pública de saúde: análise a partir do sistema H iperD ia. A rq Bras Endocrinol M etabol [ I nternet] . 2009 [ citado 2010 jul 15] ;53(1):80-6. Disponível em: http:/ / www.scielo. $\mathrm{br} / \mathrm{pdf} / \mathrm{abem} / \mathrm{v} 53 \mathrm{n} 1 / \mathrm{v} 53 \mathrm{n} 1 \mathrm{a} 12 . \mathrm{pdf}$.

16 Centers for Disease Control and Prevention. Obesity: halting the epidemic by making health easier: at a glance 2009 [ Internet] . A tlanta; 2009 [ cited 2010 Aug 10] . Availablefrom: http:/ / www.cdc.gov/ chronic disease/ resources/ publications/ AAG / obesity.htm.

\section{Endereço da autora / Dirección del autor / Author's address:}

Lílian M oura de Lima

Rua T iradentes, 451, Centro

96010-160, Pelotas, RS

E-mail:lima.lilian@gmail.com
Recebido em: 30/ 10/2010

A provado em: 18/ 04/ 2011 